\title{
Predicting typhoon-induced storm surge tide with a two-dimensional hydrodynamic model and artificial neural network model
}

\author{
W.-B. Chen ${ }^{1}$, W.-C. Liu ${ }^{2}$, and M.-H. Hsu ${ }^{3}$ \\ ${ }^{1}$ Supercomputing Research Center, National Cheng Kung University, 70101 Tainan, Taiwan \\ ${ }^{2}$ Department of Civil and Disaster Prevention Engineering, National United University, 36003 Miaoli, Taiwan \\ ${ }^{3}$ Department of Bioenvironmental Systems Engineering, National Taiwan University, 10617 Taipei, Taiwan
}

Correspondence to: W.-C. Liu (wcliu@nuu.edu.tw)

Received: 3 June 2012 - Revised: 15 November 2012 - Accepted: 15 November 2012 - Published: 21 December 2012

\begin{abstract}
Precise predictions of storm surges during typhoon events have the necessity for disaster prevention in coastal seas. This paper explores an artificial neural network (ANN) model, including the back propagation neural network (BPNN) and adaptive neuro-fuzzy inference system (ANFIS) algorithms used to correct poor calculations with a two-dimensional hydrodynamic model in predicting storm surge height during typhoon events. The two-dimensional model has a fine horizontal resolution and considers the interaction between storm surges and astronomical tides, which can be applied for describing the complicated physical properties of storm surges along the east coast of Taiwan. The model is driven by the tidal elevation at the open boundaries using a global ocean tidal model and is forced by the meteorological conditions using a cyclone model. The simulated results of the hydrodynamic model indicate that this model fails to predict storm surge height during the model calibration and verification phases as typhoons approached the east coast of Taiwan. The BPNN model can reproduce the astronomical tide level but fails to modify the prediction of the storm surge tide level. The ANFIS model satisfactorily predicts both the astronomical tide level and the storm surge height during the training and verification phases and exhibits the lowest values of mean absolute error and root-mean-square error compared to the simulated results at the different stations using the hydrodynamic model and the BPNN model. Comparison results showed that the ANFIS techniques could be successfully applied in predicting water levels along the east coastal of Taiwan during typhoon events.
\end{abstract}

\section{Introduction}

Storm surges are as a result of strong tropical storms called typhoons in the northwestern Pacific Ocean. Abnormal sea level rise due to storm surge is caused by strong winds and atmospheric pressure disturbances (You and Seo, 2009). Taiwan is located on the west side of the Pacific Ocean (Fig. 1a). The country is often subjected to severe sea states that are induced by typhoons and occur during the summer and winter seasons in either the South China Sea or the northwest Pacific Ocean near the Philippines, which result in the extensive loss of life and property. On average, Taiwan suffers three to four typhoons annually. Typhoons act more severely on the east coast than on the west coast, because typhoons typically approach Taiwan from the east. As a typhoon approaches Taiwan, its strong wind and low atmospheric pressure often cause storm surges that can result in severe damage to coastal areas, especially on the low-lying lands near river mouths because of the double effects of the river floods by typhoonbrought rains and backward uplifting seawater floods from storm surges. Therefore, it is necessary to develop a reliable model to predict the height of typhoon-induced storm surge for coastal management and hazard mitigation.

Several researchers have applied different numerical models to predict storm surges. For example, Shen et al. (2006) applied an unstructured grid numerical model (Unstructured, Tidal, Residual Intertidal, and Mudflat model - UnTRIM3D) to simulate and predict the storm tide in the Chesapeake Bay. The authors found that the use of an unstructured grid provides the flexibility to represent the complex estuarine and coastal geometry for accurately simulating storm surges. Dietsche et al. (2007) adopted the ADvance 

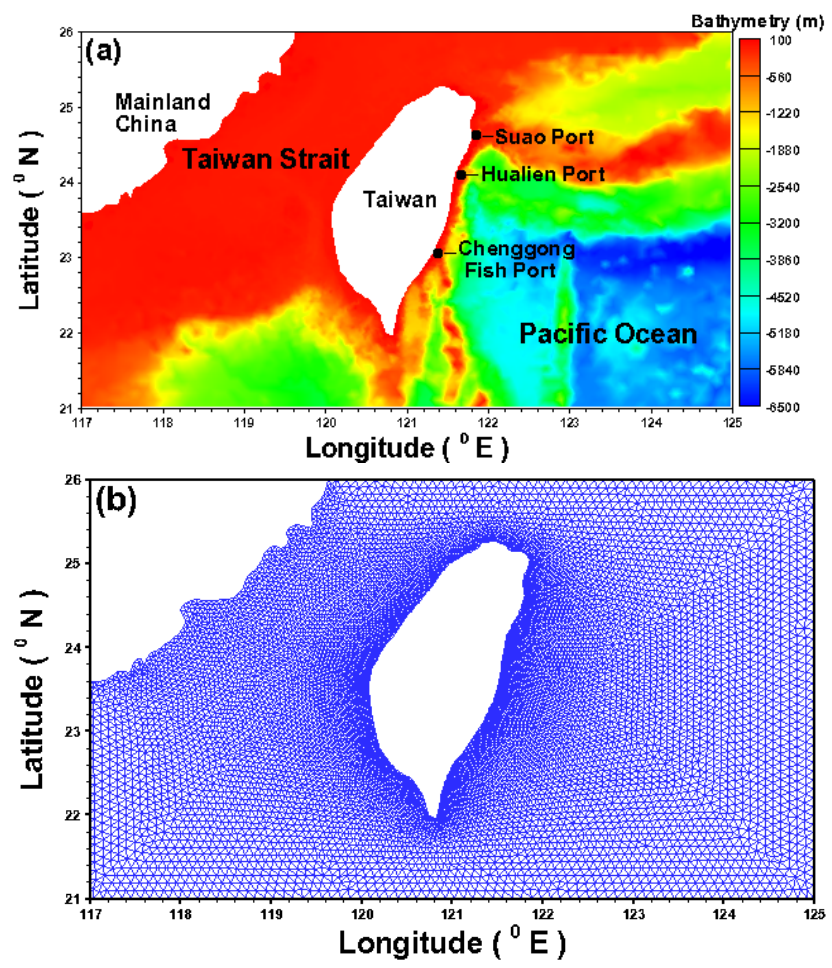

Fig. 1. (a) Bathymetric map and locations of tidal gauge stations on the east coast of Taiwan and (b) an unstructured grid in the modeling domain for the simulation.

CIRCulation (ADCIRC) model to simulate the storm surge response to two hurricane events that occurred in 1989 and 1999. Xia et al. (2008) simulated tropical cyclone storminduced surge, inundation, and coastal circulation at the Cape Fear River estuary and adjacent Long Bay using the Princeton Ocean Model (POM) with a three-level nesting approach. Etala (2009) used the nested depth-averaged numerical model for the simulation of storm surge at the Argentinean Shelf. Rego and Li (2010) studied the storm surge of Hurricane Ike along the Texas-Louisiana coast using the fully nonlinear Finite Volume Coastal Ocean Model (FVCOM) with a high-resolution unstructured mesh. You et al. (2010) compared simulated storm surges using the twodimensional operational storm surge/tide forecast system (region tide/storm surge model, RTSM, which is based on the Princeton Ocean Model), and the three-dimensional regional ocean modeling system (ROMS) using the observed data from 30 coastal tidal stations from three typhoons that struck Korea in 2007.

Recently, artificial neural network (ANN) models have been extensively applied to predict the storm surge and tide variation and to resolve the issue of nonlinear relationships. For examples, Herman et al. (2007) combined a twodimensional hydrodynamic model and neural network models to predict the tidal levels and currents in the German North Sea coast. The authors demonstrated that the approach had the capability to accurately reproduce the statistical distribution of water levels and the current in various locations and could be viewed as a reliable complementary tool. De Oliveria et al. (2009) used a neural network model to predict coastal sea level variations related to meteorological events in the southeastern coastal region of Brazil. The authors found that neural network models can efficiently predict the non-tidal residual and effectively complement the standard harmonic analysis method. You and Seo (2009) developed a cluster neural network model to predict storm surges in the Korean coastal regions. Bajo and Umgiesser (2010) developed an operational surge forecast system based on a combination of a hydrodynamic model and an artificial neural network for the city of Venice. Filippo et al. (2012) used artificial neural networks to train and translate the combined influence of meteorological and astronomical forcing to predict sea level variations.

The main objectives of this study were to apply an unstructured grid, two-dimensional hydrodynamic model (ADCIRC-2DDI) to simulate the astronomical tide level and storm surge height along the east coast of Taiwan. Two artificial neural network models were subsequently adopted to improve the calculations of the hydrodynamic model. Three quantitative statistical measures, i.e. the mean absolute error, root-mean-square error, and peak error, were employed to evaluate the prediction of the tide and storm surges under the five typhoon events by a hydrodynamic model and an ANN model, including the back propagation neural network (BPNN) and adaptive neuro-fuzzy inference system (ANFIS) techniques.

\section{Hydrodynamic model}

\subsection{Storm surge model}

The water surface elevations and circulation patterns are generated using the ADCIRC-2DDI, which is a vertically averaged two-dimensional, fully nonlinear, hydrodynamic model (Luettich, 1992). ADCIRC-2DDI uses the mass and momentum conservation equations with the hydrostatic pressure approximation. In the model, the baroclinic terms are neglected and the hybrid bottom friction formulation is used. The lateral diffusion/dispersion terms are enabled, which leads to the equality laws in primitive, nonconservative form expressed in a spherical coordinate system (Kolar et al., 1994):

$$
\begin{aligned}
& \frac{\partial \zeta}{\partial t}+\frac{1}{R \cos \phi}\left[\frac{\partial U H}{\partial \lambda}+\frac{\partial(V H \cos \phi)}{\partial \phi}\right]=0 \\
& \frac{\partial U}{\partial t}+\frac{1}{R \cos \phi} U \frac{\partial U}{\partial \lambda}+\frac{1}{R} V \frac{\partial U}{\partial \phi}-\left(\frac{\tan \phi}{R} U+f\right) V \\
& =-\frac{1}{R \cos \phi} \frac{\partial}{\partial \lambda}\left[\frac{p_{\mathrm{s}}}{\rho_{0}}+g(\zeta-\alpha \eta)\right]+\frac{1}{H} M_{\lambda}+\frac{\tau_{\mathrm{s} \lambda}}{\rho_{0} H}-\frac{\tau_{\mathrm{b} \lambda}}{\rho_{0} H}
\end{aligned}
$$




$$
\begin{aligned}
& \frac{\partial V}{\partial t}+\frac{1}{R \cos \phi} U \frac{\partial V}{\partial \lambda}+\frac{1}{R} V \frac{\partial V}{\partial \phi}+\left(\frac{\tan \phi}{R} U+f\right) U \\
& =-\frac{1}{R} \frac{\partial}{\partial \phi}\left[\frac{p_{\mathrm{s}}}{\rho_{0}}+g(\zeta-\alpha \eta)\right]+\frac{1}{H} M_{\phi}+\frac{\tau_{\mathrm{s} \phi}}{\rho_{0} H}-\frac{\tau_{\mathrm{b} \phi}}{\rho_{0} H}
\end{aligned}
$$

where the vertically averaged momentum dispersion in the longitudinal and the latitudinal directions can be expressed as follows (Kolar and Gray, 1990):

$$
M_{\lambda, \phi}=\frac{E_{\mathrm{h} 2}}{R^{2}}\left[\frac{1}{\cos ^{2} \phi} \frac{\partial^{2}(U, V) H}{\partial \lambda^{2}}+\frac{\partial^{2}(U, V) H}{\partial \phi^{2}}\right],
$$

and $t=$ time; $\lambda, \phi=$ degrees longitude (east of Greenwich is positive) and latitude (north of the Equator is positive), respectively; $U, V=$ the depth-averaged velocity in the longitudinal and latitudinal directions, respectively; $H=$ the total height of the vertical water column $(=h+\zeta) ; h=$ the bathymetric depth, relative to mean sea level (MSL); $\zeta=$ the free surface elevation, relative to MSL; $R=$ the radius of the Earth; $f=2 \Omega \sin \phi=$ the Coriolis parameter; $\Omega=$ the angular speed of the Earth; $p_{\mathrm{s}}=$ the atmospheric pressure at the free surface; $\rho_{0}=$ the reference density of water; $g=$ the acceleration due to gravity; $\alpha=$ the effective Earth elasticity factor; $E_{\mathrm{h} 2}=$ the horizontal eddy viscosity; $\tau_{\mathrm{s} \lambda}, \tau_{\mathrm{s} \phi}=$ the applied free surface stress in the longitudinal and the latitudinal directions, respectively; $\tau_{\mathrm{b} \lambda}, \tau_{\mathrm{b} \phi}=$ the bottom shear stresses in the longitudinal and the latitudinal directions, respectively, and $\eta=$ the Newtonian equilibrium tide potential.

In the ADCIRC-2DDI model, the hybrid bottom friction formulation is used when the wetting and drying of elements is executed, because this expression results in being highly dissipative as the water depth becomes small (Grenier et al., 1995).

The bottom friction in the model is expressed as

$\tau_{\mathrm{b} \lambda}=U \tau_{*}$ and $\tau_{\mathrm{b} \phi}=V \tau_{*}$.

The hybrid bottom friction $\left(\tau_{*}\right)$ formulation, which provided a depth-dependent bottom friction coefficient, can be defined by the following formula:

$\tau_{*}=\frac{C_{\mathrm{f}}\left(U^{2}+V^{2}\right)^{1 / 2}}{H}$,

where $C_{\mathrm{f}}=C_{\mathrm{f} \min }\left[1+\left(\frac{H_{\mathrm{break}}}{H}\right)^{\theta}\right]^{\gamma / \theta}=$ the bottom drag coefficient. The unit of $C_{\mathrm{f}}$ is $\mathrm{kg} \mathrm{m}^{-2} ; C_{\mathrm{fmin}}=$ the minimum bottom drag coefficient that is approached in deep water when the hybrid bottom friction formulation reverts to a standard quadratic bottom friction function; $H_{\text {break }}=$ the break depth to determine if the hybrid bottom friction formulation will behave as a standard bottom friction function or increase with depth similar to a Manning's type bottom friction function; and $\theta$ and $\gamma=$ dimensionless parameters which are given in Sect. 4.2.

ADCIRC-2DDI is Courant-limited algorithmically due to its explicit feature, and it is also limited by the propagation speed of any wetting fronts. The Courant number is less than 0.5 to maintain numerical stability. More details of ADCIRC2DDI model can be found in Luettich et al. (1992), Kolar et al. (1994), and Westerink et al. (1994).

\subsection{Global tidal model}

A large fraction of the variance in many oceanographic variables is due to tides. To simulate tidal propagation in the storm surge model, the driving tidal forces at the open boundaries are necessary. In the present study, a global ocean tidal model that was developed by Oregon State University, which is the TOPEX/Poseidon Global Inverse Solution (TPXO), is used to specify the open boundaries of ADCIRC for simulating tidal propagation. TPXO is the current version of a global model of ocean tides, and it incorporates data from the TOPEX/Poseidon satellite. The tides are provided as complex amplitudes of the Earth-relative sea-surface elevation for eight primary $\left(M_{2}, S_{2}, N_{2}, K_{2}, K_{1}, O_{1}, P_{1}\right.$, and $\left.Q_{1}\right)$ and two long-period $\left(M_{\mathrm{f}}\right.$ and $\left.M_{\mathrm{m}}\right)$ harmonic constituents. The methods used to compute the model are described in detail by Egbert et al. (1994) and Egbert and Erofeeva (2002).

\subsection{Cyclone model}

The meteorological driving forces underlying storm surges consist of wind stress and an atmospheric pressure gradient. Therefore, determining the wind field and the pressure field of a tropical cyclone is indispensable for conducting cyclone surge calculations.

In the present study, the atmospheric pressure field derived from cyclostrophic flow can be expressed as follows (Jelesnianski, 1965):

$$
\begin{aligned}
& P_{\mathrm{r}}=P_{\mathrm{c}}+\frac{1}{4}\left(P_{n}-P_{\mathrm{c}}\right)\left(\frac{r}{R_{\max }}\right)^{3}, \text { for } 0 \leq r \leq R_{\max } \\
& P_{\mathrm{r}}=P_{n}-\frac{3}{4}\left(P_{n}-P_{\mathrm{c}}\right)\left(\frac{r}{R_{\max }}\right)^{-1}, \text { for } r>R_{\max },
\end{aligned}
$$

where $P_{\mathrm{c}}=$ the pressure at the typhoon center; $P_{n}=$ the ambient pressure and was assumed to be equal to $1013 \mathrm{hPa} ; r=$ the radius which is the distance from the typhoon center; and $R_{\max }=$ the radius of the maximum wind. Following Graham and Nunn (1959), $R_{\max }$ is represented by the following equation:

$$
\begin{aligned}
& R_{\max }=28.52 \tanh [0.0873(\phi-28)] \\
& +12.22 / \exp \left[\left(1013-P_{\mathrm{c}}\right) / 33.86\right]+0.2 V_{\mathrm{f}}+32.77,
\end{aligned}
$$

where $\phi$ is the latitude of the typhoon center and $V_{\mathrm{f}}$ is the forward speed of the typhoon.

The wind field comprises a rotational and a translational component. At a distance, $r$, from the center of the typhoon, the rotational wind speed, $V_{\mathrm{r}}$, is described by the following equation (Young and Sobey, 1981): 
$V_{\mathrm{r}}=V_{\max }\left(r / R_{\max }\right)^{7} \cdot \exp \left[7\left(1-r / R_{\max }\right)\right]$ for $r<R_{\max }$

$V_{\mathrm{r}}=V_{\max } \cdot \exp \left[\left(0.0025 \mathrm{R}_{\max }+0.05\right)\left(1-r / R_{\max }\right)\right]$

for $r \geq R_{\max }$,

where $V_{\max }=6.3\left(1013.0-P_{0}\right)^{1 / 2}$.

The translational component of wind field, $V_{\mathrm{t}}$, can be expressed as

$V_{\mathrm{t}}=-0.5 V_{\mathrm{f}} \cdot(-\cos \varphi)$,

where $V_{\mathrm{f}}$ is the moving speed of typhoon and $\varphi$ is the angle between the radial arm and the line of maximum wind.

The resultant wind speed $\left(V_{\mathrm{w}}\right)$ inside the typhoon is given as

$V_{\mathrm{w}}=V_{\mathrm{r}}+V_{\mathrm{t}}$.

\subsection{Geological structure of the model}

The computational domain in the Asian marginal seas and the western Pacific Ocean includes the region within the longitudes $117^{\circ} \mathrm{E}$ to $125^{\circ} \mathrm{E}$ and the latitudes $21^{\circ} \mathrm{N}$ to $28^{\circ} \mathrm{N}$. The digital terrain model (DTM) bathymetric data were obtained from the Global Topography data bank of the University of California, San Diego, and from the Ocean Data Bank of the National Science Council, Taiwan. Figure 1a shows the bathymetric map and the locations of the tidal gauge stations on the east coast of Taiwan. To save computational time and fit the coastline, coarse grids were generated in coastal seas, whereas fine grids were used in shallow areas close to the coastline. The modeling domain consisted of 18543 unstructured triangular elements and 9560 nodes (Fig. 1b). To fit the raw bathymetric data and simply define the open boundary conditions at corner points, rectangular area (see Fig. 1b) was adopted in the modeling domain. For this model grid, a time step of $\Delta t=20 \mathrm{~s}$ was used in simulations, which was intended to maintain the Courant number less than 0.5 and showed no sign of instability.

\section{Artificial neural network (ANN) models}

In the present study, two ANN models, including BPNN and ANFIS, were introduced. The algorithms of these two ANN models are described as follows.

\subsection{Back propagation neural network (BPNN)}

A back propagation neural network (BPNN) was used to amend the simulated water levels with the two-dimensional hydrodynamic model for precise predictions. The BPNN, which was proposed by Rumelhart et al. (1986), is a multiplelayer network with nonlinear differentiable transfer functions including input layer, hidden layer, and output layer.

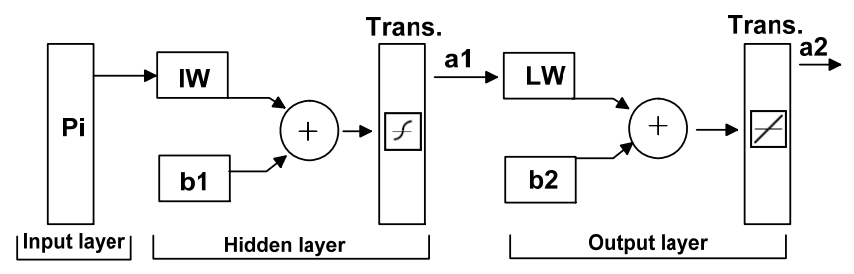

Fig. 2. Architecture of the back propagation neural network (BPNN).

Figure 2 shows the architecture of the BPNN. In this figure, "Trans." represents the transfer function. The connections between input and hidden layer neurons and between hidden and output layer neurons can be illustrated by the Eqs. (11) and (12), respectively.

$a 1=f(\mathrm{IW} \times \mathrm{Pi}+b 1)$

$a 2=f(\mathrm{LW} \times a 1+b 2)$

where $a 1$ and $a 2$ are the values of the hidden layer and the output layer, respectively; $\mathrm{Pi}$ is the input vector; IW and $b 1$ are weights and biases, respectively, between the input layer and the hidden layer; and LW and $b 2$ are weight and biases, respectively, between the hidden layer and the output layer.

A hyperbolic tangent sigmoid transfer function in Eq. (13) is used in the hidden layer.

$f(x)=\frac{2}{\left(1+e^{-2 x}\right)-1}$

And a linear transfer function in Eq. (14) is applied in the output layer.

$f(x)=x$

To scale the inputs and targets, the normalized equation is often used in Eq. (15) so that the data always fall within a specified range.

$Y_{\mathrm{N}}=\left(y_{\text {max }}-y_{\text {min }}\right) \times\left(\frac{x_{i}-x_{\text {min }}}{x_{\text {max }}-x_{\text {min }}}\right)+y_{\text {min }}$,

where $Y_{\mathrm{N}}$ is the value after normalization; $x_{\min }$ and $x_{\max }$ denote the minimum and maximum of the data, respectively; and $y_{\min }$ and $y_{\max }$ are taken as -1 and 1 . The performance of the network can be evaluated according to the mean square error (MSE) in Eq. (16):

$\mathrm{MSE}=\frac{\sum_{i=1}^{N}\left(a_{2}-y_{i}\right)^{2}}{N}$,

where $y_{i}$ represents the observed value and $N$ is the total number of data. When the leaning performance (MSE) is less than a tolerance value, the iteration terminates. The tolerance value, $10^{-3}$, was adopted in this model. 
In the present study, BPNN updates weight and bias values according to the Fletcher-Powell conjugate gradient (Fletcher and Powell, 1963). The BPNN model was performed in MATLAB where the Fletcher-Powell conjugate gradient technique is applicable in the Neural Network Toolbox.

\subsection{Adaptive neuro-fuzzy inference system (ANFIS)}

ANFIS is a multilayer feed-forward neural network where each node executes a particular function based on the incoming signals. The symbols with square and circle nodes are applied to stand for different properties of adaptive learning (see Fig. 3). In order to yield the desired input-output characteristics, the parameters of adaptive learning are updated according to the gradient learning rules. ANFIS learns the rules and membership functions from data sets (Jang, 1993). The primary weakness of the ANFIS model is consuming much time for the training and parameter determination. In this study, ANFIS takes about 20 min to complete the training and verification, while the BPNN spends $10 \mathrm{~s}$ only.

Essentially, the fuzzy inference system has two input variables, $x_{1}$ and $x_{2}$, and one output variable, $y$. The assumption is that the rule base includes two fuzzy if-then rules with a first-order Sugeno fuzzy model (Takagi and Sugeno, 1985):

Rule 1: If $x_{1}$ is $A_{1}$ and $x_{2}$ is $B_{1}$, then $y_{1}=p_{1} x_{1}+q_{1} x_{2}+r_{1}$

Rule 2: If $x_{1}$ is $A_{2}$ and $x_{2}$ is $B_{2}$, then $y_{2}=p_{2} x_{1}+q_{2} x_{2}+r_{2}$, where $A_{i}$ and $B_{i}$ are the fuzzy sets and $p_{i}, q_{i}$ and $r_{i}$ are the design parameters that will be determined during the training and verification processes. The architecture of ANFIS is shown in Fig. 3, where circles represent fixed nodes and squares indicate adaptive nodes. A brief introduction of the ANFIS model follows.

Input nodes (Layer 1): Each node $i$ in this layer is a square node (see Fig. 3). The node function can be expressed as

$O_{1, i}=\mu_{A_{i}}\left(x_{1}\right), i=1,2$

$O_{1, i}=\mu_{B_{i-2}}\left(x_{2}\right), i=3,4$

where $x_{1}$ and $x_{2}$ are the inputs to node $i ; A_{i}$ and $B_{i}$ are the linguistic labels; and $\mu_{A_{i}}$ and $\mu_{B_{i-2}}$ indicate the membership function for the $A_{i}$ and $B_{i}$ linguistic labels, respectively. In this study, the following bell-shaped membership function is applied:

$\mu_{A_{i}}=\frac{1}{1+\left|\frac{x_{1}-c_{i}}{a_{i}}\right|^{2 b_{i}}} \mu_{B_{i-2}}=\frac{1}{1+\left|\frac{x_{2}-c_{i}}{a_{i}}\right|^{2 b_{i}}}$,

where $a_{i}, b_{i}$ and $c_{i}$ are the parameter sets which are the premise parameters in this layer.

Rule nodes (Layer 2): In this layer, every node indicates a circle node labeled $M$ (Fig. 3). The outputs of this layer, named firing strengths $\left(O_{2, i}\right)$, are the products of the corresponding degrees which are obtained from input layer (Layer 1).

$O_{2, i}=w_{i}=\mu_{A_{i}}\left(x_{1}\right) \times \mu_{B_{i}}\left(x_{1}\right), i=1,2$

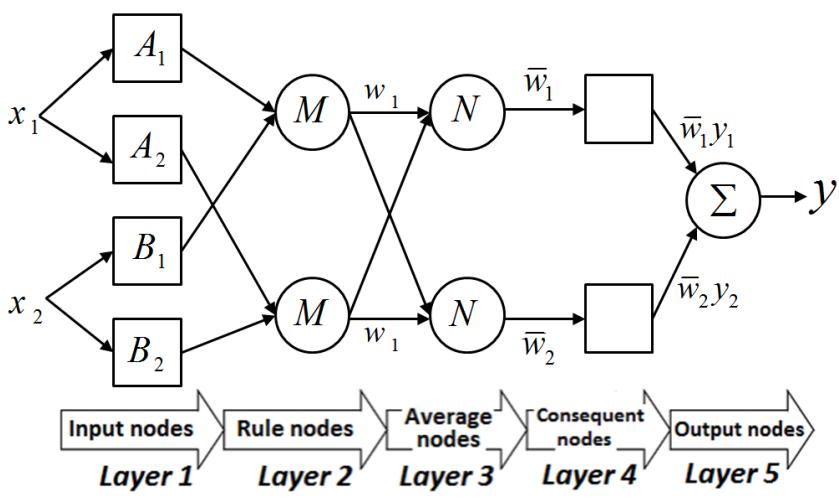

Fig. 3. Architecture of the adaptive network-based fuzzy interface system (ANFIS).

Average nodes (Layer 3): in this layer, every node presents a circle node labeled $N$ (Fig. 3). This layer includes fixed nodes which calculate the ratio of the firing strengths of the rules:

$O_{3, i}=\bar{w}_{i}=\frac{w_{i}}{w_{1}+w_{2}}, i=1,2$.

Consequent nodes (Layer 4): The nodes in this layer are adaptive. The output of each node, which is a first-order polynomial, is the product of the normalized firing strength. The output can be illustrated as

$O_{4, i}=\bar{w}_{i} y_{i}=\bar{w}_{i}\left(p_{i} x+q_{i} y+r_{i}\right), i=1,2$.

The parameters, $p_{i}, q_{i}$, and $r_{i}$, in this layer indicate the coefficients of linear combination.

Output nodes (Layer 5): The single node calculates the overall output by integrating all of the input signals.

$O_{5,1}=\sum_{i=1}^{2} \bar{w}_{i} y_{i}=\frac{\sum_{i=1}^{2} w_{i} y}{w_{1}+w_{2}}$

The detailed algorithms of ANFIS technique can be found in Jang et al. (1993) and Nayak et al. (2004).

\subsection{Indices of simulation performance}

To evaluate the performance of the two-dimensional hydrodynamic model and the ANN model, three criteria were adopted to compare the predicted results and the observational data: the mean absolute error (MAE), root-meansquare error (RMSE), and peak error (PE). These criteria are defined by the following equations: 
Table 1. The performance of the two-dimensional hydrodynamic model, BPNN model, and ANFIS model for predicting water level during the training (calibration) phase at different stations.

\begin{tabular}{|c|c|c|c|c|c|c|c|c|c|}
\hline \multirow{2}{*}{ Method } & \multicolumn{3}{|c|}{ Suao Port } & \multicolumn{3}{|c|}{ Hualien Port } & \multicolumn{3}{|c|}{ Chenggong Fish Port } \\
\hline & $\begin{array}{l}\mathrm{PE} \\
(\%)\end{array}$ & $\begin{array}{r}\text { MAE } \\
(\mathrm{m})\end{array}$ & $\begin{array}{r}\text { RMSE } \\
(\mathrm{m})\end{array}$ & $\begin{array}{l}\mathrm{PE} \\
(\%)\end{array}$ & $\begin{array}{r}\text { MAE } \\
(\mathrm{m})\end{array}$ & $\begin{array}{r}\text { RMSE } \\
(\mathrm{m})\end{array}$ & $\begin{array}{l}\mathrm{PE} \\
(\%)\end{array}$ & $\begin{array}{r}\text { MAE } \\
(\mathrm{m})\end{array}$ & $\begin{array}{r}\text { RMSE } \\
(\mathrm{m})\end{array}$ \\
\hline $\begin{array}{l}\text { Calibration with two-dimensional } \\
\text { hydrodynamic model }\end{array}$ & 23.52 & 0.17 & 0.23 & 21.57 & 0.20 & 0.25 & 23.28 & 0.24 & 0.29 \\
\hline Training with BPNN model & -15.19 & 0.15 & 0.20 & -17.04 & 0.21 & 0.26 & -23.43 & 0.16 & 0.21 \\
\hline Training with ANFIS model & 1.91 & 0.11 & 0.16 & 1.24 & 0.16 & 0.21 & -0.82 & 0.11 & 0.15 \\
\hline
\end{tabular}

Note: PE represents peak error; MAE represents mean absolute error; and RMSE represents root-mean-square error.

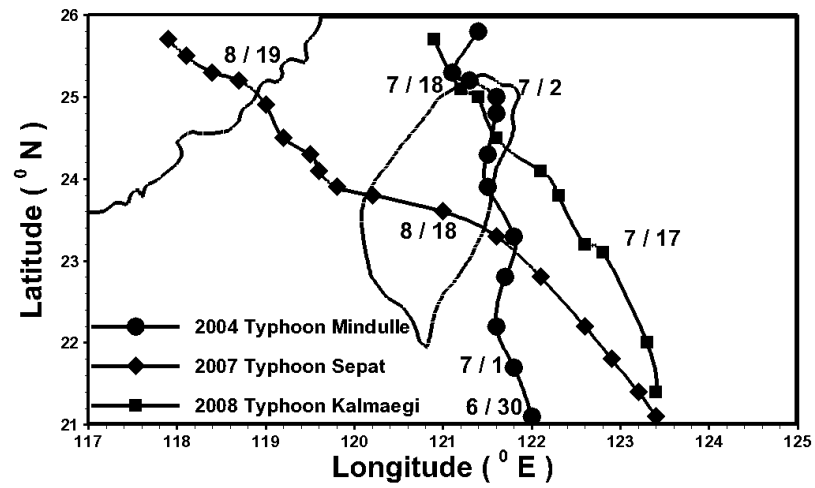

Fig. 4. Tracks of Typhoon Mindulle (2004), Typhoon Sepat (2007), and Typhoon Kalmaegi (2008) for hydrodynamic model calibration (ANN training).

$$
\begin{aligned}
\text { MAE } & =\frac{1}{N} \sum_{i=1}^{N}\left|\left(Y_{\mathrm{m}}\right)_{i}-\left(Y_{\mathrm{o}}\right)_{i}\right| \\
\mathrm{RMSE} & =\sqrt{\frac{1}{N} \sum_{i=1}^{N}\left[\left(Y_{\mathrm{m}}\right)_{i}-\left(Y_{\mathrm{o}}\right)_{i}\right]^{2},} \\
\mathrm{PE} & =\frac{Y_{\text {m,peak }}-Y_{\text {o,peak }}}{Y_{\text {o,peak }}} \times 100 \%
\end{aligned}
$$

where $N$ is the total number of data; $Y_{\mathrm{m}}$ is the predicted water level; $Y_{\mathrm{o}}$ is the observational water level; $Y_{\mathrm{m} \text {,peak }}$ is the predicted peak water level; and $Y_{\text {o,peak }}$ is the observational peak water level. The negative PE represents that the model underpredicts the peak observed water level, while the positive PE means that the model overpredicts the peak water level.

\section{Model validity}

Five data sets were used to determine the practical accuracy of the model and to ascertain its predictive capabilities. Typhoon Mindulle (2004), Typhoon Sepat (2007), and Typhoon Kalmaegi (2008) (792 hourly water level data) were

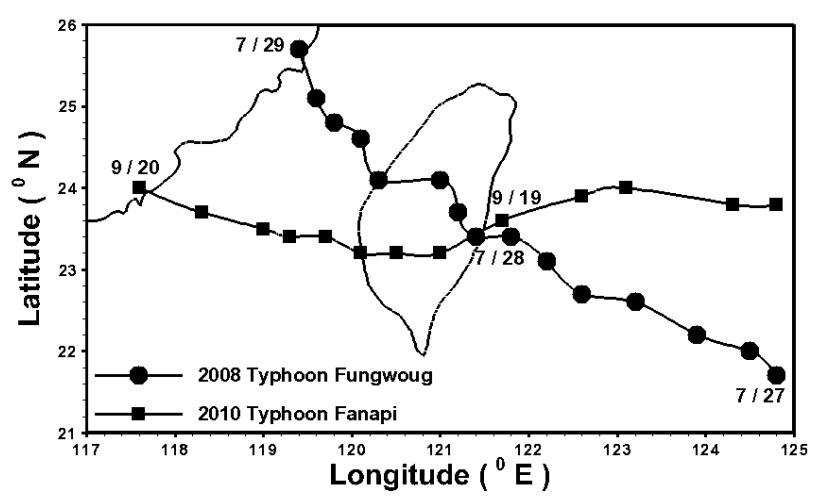

Fig. 5. Tracks of Typhoon Fungwoug (2008) and Typhoon Fanapi (2010) for hydrodynamic model verification (ANN verification).

employed for hydrodynamic model calibration (ANN model training), while Typhoon Fungwoug (2008) and Typhoon Fanapi (2010) (480 hourly water level data) were adopted for hydrodynamic model verification (ANN model verification). Figures 4 and 5 show the paths of these five typhoon events for model calibration (ANN training) and model verification (ANN verification).

\subsection{Hydrodynamic model calibration and ANN model training}

The terminologies for model comparisons, such as the training and verification phases with the ANN model (BPNN and ANFIS approaches), were used as analogs for the calibration and validation with the two-dimensional hydrodynamic (ADCIRC) model, respectively. The scatter plot of simulated and observed water level using two-dimensional hydrodynamic model is shown in Fig. 6. The hydrodynamic model obviously underpredicts storm surge height. Table 1 shows the PE, MAE, and RMSE for the model calibration. The maximum PE, MAE, and RMSE values for Chenggong Fish Port are $23.28 \%, 0.24 \mathrm{~m}$ and $0.29 \mathrm{~m}$, respectively.

Due to the failure in simulating surge height of the two-dimensional hydrodynamic model as the typhoon 

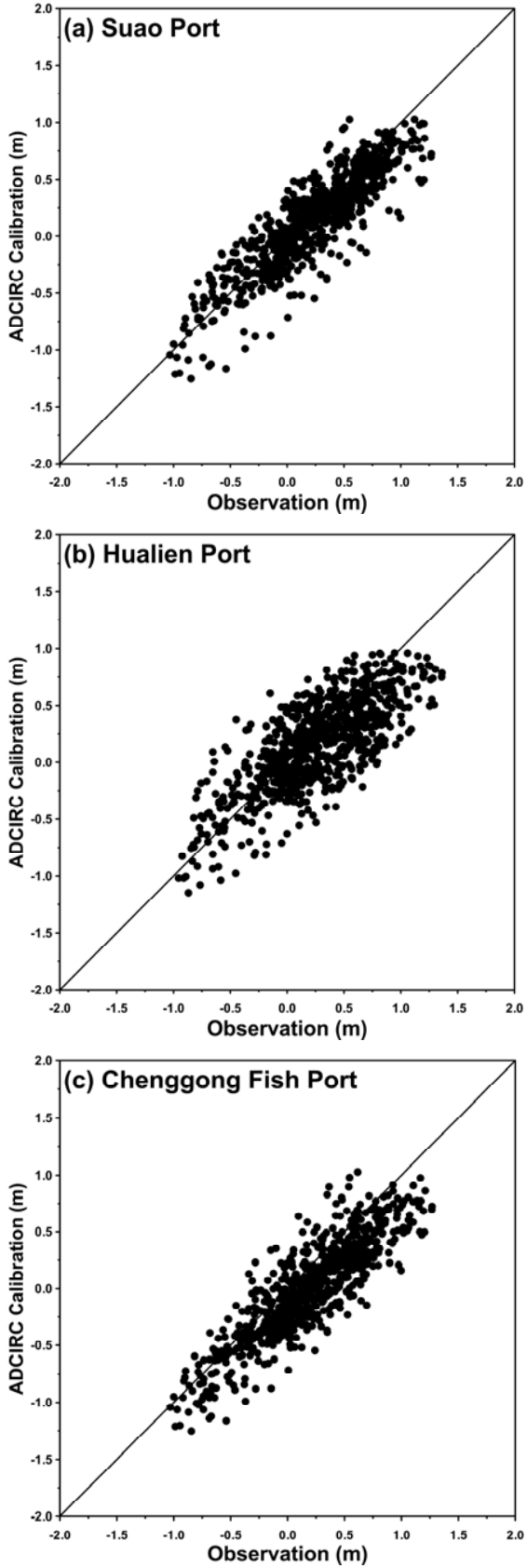

Fig. 6. Scatter plot of simulated and observed water level using two-dimensional hydrodynamic model for the model calibration at (a) Suao Port, (b) Hualien Port, and (c) Chenggong Fish Port.

approached Taiwan, ANN models including BPNN and ANFIS were adopted to improve the water level calculations of the two-dimensional hydrodynamic model. BPNN structures for the Suao Port serve as an example and are shown in Fig. 7. The input layer includes wind speed, wind direction, and air pressure generated from cyclone model (CM) as well as the simulated water level at the Suao Port using the

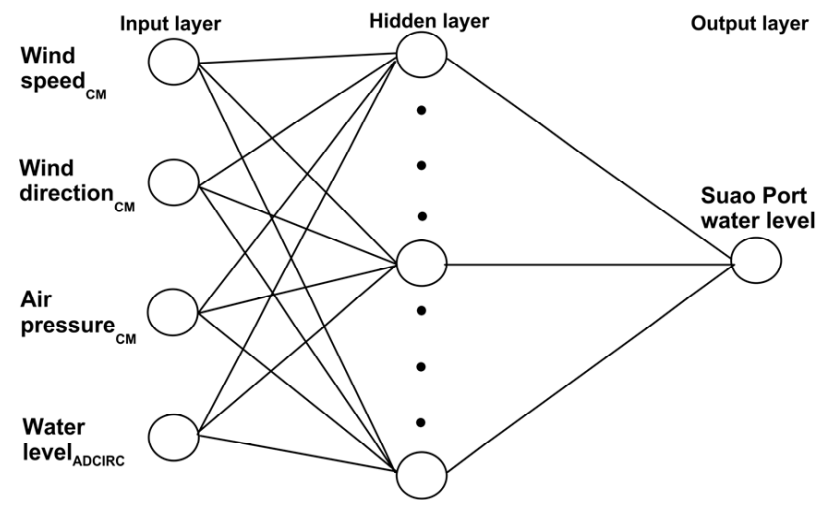

Fig. 7. BPNN structures for Suao Prot. Notably, CM represents the simulated data from cyclone model, and ADCIRC represents the simulated water level, which was obtained from the twodimensional hydrodynamic model (ADCIRC).
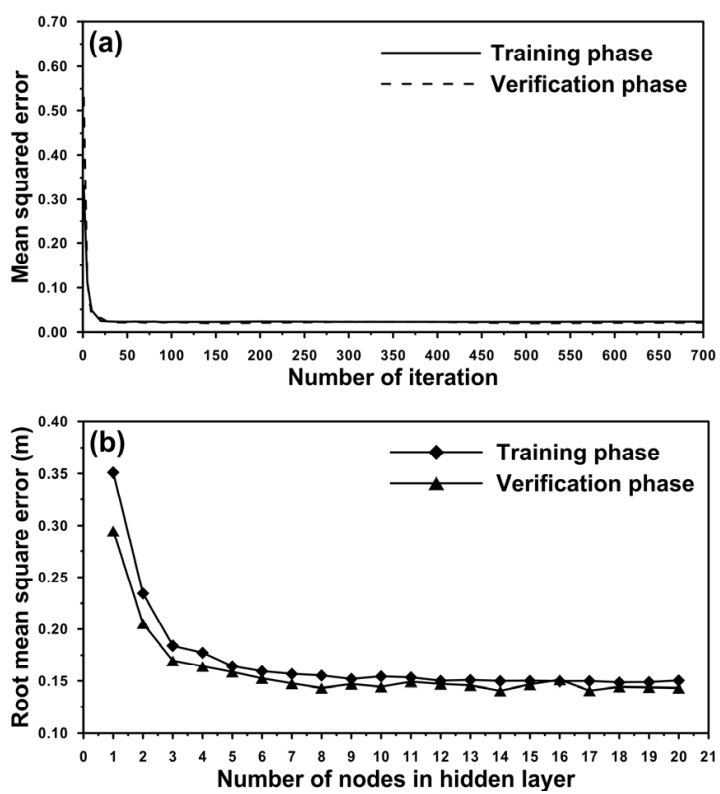

Fig. 8. (a) Variation in the mean square error (MSE) with iterations and (b) the effect of the number of nodes in the hidden layer on the root-mean-square error (RMSE) for Suao Port.

two-dimensional hydrodynamic model, and the output layer is the predicted water level at the Suao Port.

Figure 8a shows relationship between the mean square error (MSE) and the number of iterations for the Suao Port during the training and verification phases. The MSE did not significantly change when the number of iterations exceeded 300. Therefore, 400 iterations were adopted for BPNN training and verification. In this study, a learning rate of 0.01 and a momentum coefficient of 0.3 were used. Figure $8 b$ presents the influence of different number of hidden nodes on the RMSE for BPNN training and verification phases. To yield the optimal number of nodes in the hidden layer, 16 hidden 
Table 2. The parameters used in the BPNN model.

\begin{tabular}{lrrr}
\hline Parameters & $\begin{array}{r}\text { Suao } \\
\text { Port }\end{array}$ & $\begin{array}{r}\text { Hualien } \\
\text { Port }\end{array}$ & $\begin{array}{r}\text { Chenggong } \\
\text { Fish Port }\end{array}$ \\
\hline Learning rate & 0.01 & 0.01 & 0.01 \\
Momentum & 0.3 & 0.3 & 0.3 \\
Iteration & 400 & 400 & 400 \\
Input nodes & 4 & 4 & 4 \\
Hidden nodes & 16 & 16 & 17 \\
Output nodes & 1 & 1 & 1 \\
\hline
\end{tabular}

nodes in the BPNN model were chosen during the training and verification phases. Table 2 shows the parameters used in the BPNN model.

The statistical errors of performance with the BPNN model for predicting water level during the training phase are shown in Table 1. Overall, the BPNN model does not significantly improve the prediction of water level. The MAE values at Suao Port and Hualien Port for the BPNN model are greater than that for the two-dimensional hydrodynamic model. The maximum values of PE, MAE, and RMSE are $-23.43 \%$ at Chenggong Fish Port, $0.21 \mathrm{~m}$, and $0.26 \mathrm{~m}$ at Hualien Port, respectively.

Table 1 also shows the performance evaluations for predicting the water levels at different stations using the ANFIS model. The prediction of water level with the ANFIS model is better than that with the two-dimensional hydrodynamic model and the BPNN model. This table indicates that the PE, MAE, and RMSE values at Suao Port, Hualien Port, and Chenggong Fish Port of the ANFIS model are less than those of the two-dimensional hydrodynamic model and the BPNN model. The maximum PE, MAE, and RMSE values for ANFIS training phase are $1.91 \%, 0.16 \mathrm{~m}$, and $0.21 \mathrm{~m}$, respectively. A comparison of the results indicates that the ANFIS technique is successfully used in predicting the astronomical tide level and storm surge height.

\subsection{Hydrodynamic model verification and ANN model verification}

The verification results with the two-dimensional hydrodynamic model for simulating the water levels at different locations during the period of Typhoon Fanapi (2010) are illustrated in Fig. 9. Due to space limitations, we only show the modeling results during Typhoon Fanapi (2010). The numerical model reproduces the astronomical tide level, but it fails to simulate water levels when the storm surge occurred on 19 September 2010. The hydrodynamic model underpredicts the storm surge height. Table 3 shows the PE, MAE, and RMSE for model verification. The results indicate that the PE, MAE, and RMSE values at Suao Port and Hualien Port are less than those at Chenggong Fish Port.

Through the model calibration and verification procedures, the horizontal eddy viscosity parameter in Eq. (4) is
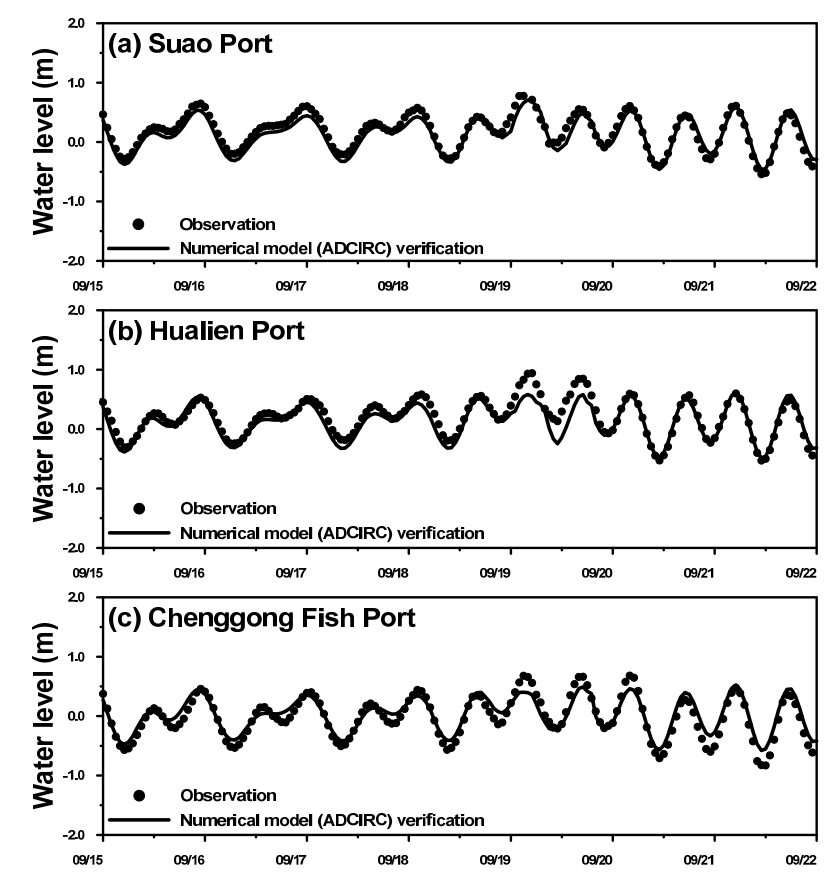

Fig. 9. Comparison of the observed (circle mark) and simulated (black line) water levels for the two-dimensional hydrodynamic model verification at (a) Suao Port, (b) Hualien Port, and (c) Chenggong Fish Port during Typhoon Fanapi (2010).

$5.0 \mathrm{~m}^{2} \mathrm{~s}^{-1}$. A constant minimum bottom friction coefficient of $C_{\mathrm{fmin}}=0.003$, break depth of $H_{\mathrm{break}}=10 \mathrm{~m}$, and two dimensionless parameters of $\theta=10$ and $\gamma=1 / 3$ were used.

Figure 10 shows the prediction of water level at different stations by the BPNN model during the verification phase. The BPNN model fails to modify the prediction of storm surge height at Hualien Port and Chenggong Fish Port compared to the simulated results of the two-dimensional hydrodynamic model (Fig. 9). Because BPNN model predicts tide and storm surge based upon the inputs, a large peak error at the Chenggong Fish Port can be taken into account - a bad prediction with BPNN model (Fig. 10c and Table 3). The PE, MAE, and RMSE values at Suao Port, Hualien Port, and Chenggong Fish Port with the BPNN model are higher than with the two-dimensional hydrodynamic model (see Table 3). Table 3 also indicates that the maximum PE, MAE, and RMSE values are $-43.89 \%, 0.23 \mathrm{~m}$, and $0.28 \mathrm{~m}$, respectively, for the BPNN verification phase.

Figure 11 presents the prediction of water level at different stations by the ANFIS model during the verification phase. The simulated water level with the ANFIS model is better than with the two-dimensional hydrodynamic model and the BPNN model. It indicates that the ANFIS model satisfactorily mimics the observed astronomical tide level and surge height. Table 3 also indicates that the PE, MAE, and RMSE values at Suao Port, Hualien Port, and Chenggong 
Table 3. The performance of the two-dimensional hydrodynamic model, BPNN model, and ANFIS model for predicting water level during the verification phase at different stations.

\begin{tabular}{|c|c|c|c|c|c|c|c|c|c|}
\hline \multirow{2}{*}{ Method } & \multicolumn{3}{|c|}{ Suao Port } & \multicolumn{3}{|c|}{ Hualien Port } & \multicolumn{3}{|c|}{ Chenggong Fish Port } \\
\hline & $\begin{array}{l}\mathrm{PE} \\
(\%)\end{array}$ & $\begin{array}{r}\text { MAE } \\
(\mathrm{m})\end{array}$ & $\begin{array}{r}\text { RMSE } \\
(\mathrm{m})\end{array}$ & $\begin{array}{l}\mathrm{PE} \\
(\%)\end{array}$ & $\begin{array}{r}\text { MAE } \\
(\mathrm{m})\end{array}$ & $\begin{array}{r}\text { RMSE } \\
(\mathrm{m})\end{array}$ & $\begin{array}{r}\mathrm{PE} \\
(\%)\end{array}$ & $\begin{array}{r}\text { MAE } \\
(\mathrm{m})\end{array}$ & $\begin{array}{r}\text { RMSE } \\
(\mathrm{m})\end{array}$ \\
\hline $\begin{array}{l}\text { Verification with two-dimensional } \\
\text { hydrodynamic model }\end{array}$ & -7.16 & 0.13 & 0.16 & -1.49 & 0.17 & 0.20 & -20.30 & 0.19 & 0.23 \\
\hline Verification with BPNN model & -36.10 & 0.17 & 0.21 & -32.94 & 0.21 & 0.25 & -43.89 & 0.23 & 0.28 \\
\hline Verification with ANFIS model & -2.16 & 0.07 & 0.10 & 0.61 & 0.10 & 0.14 & -13.77 & 0.11 & 0.16 \\
\hline
\end{tabular}

Note: PE represents peak error; MAE represents mean absolute error; and RMSE represents root-mean-square error.
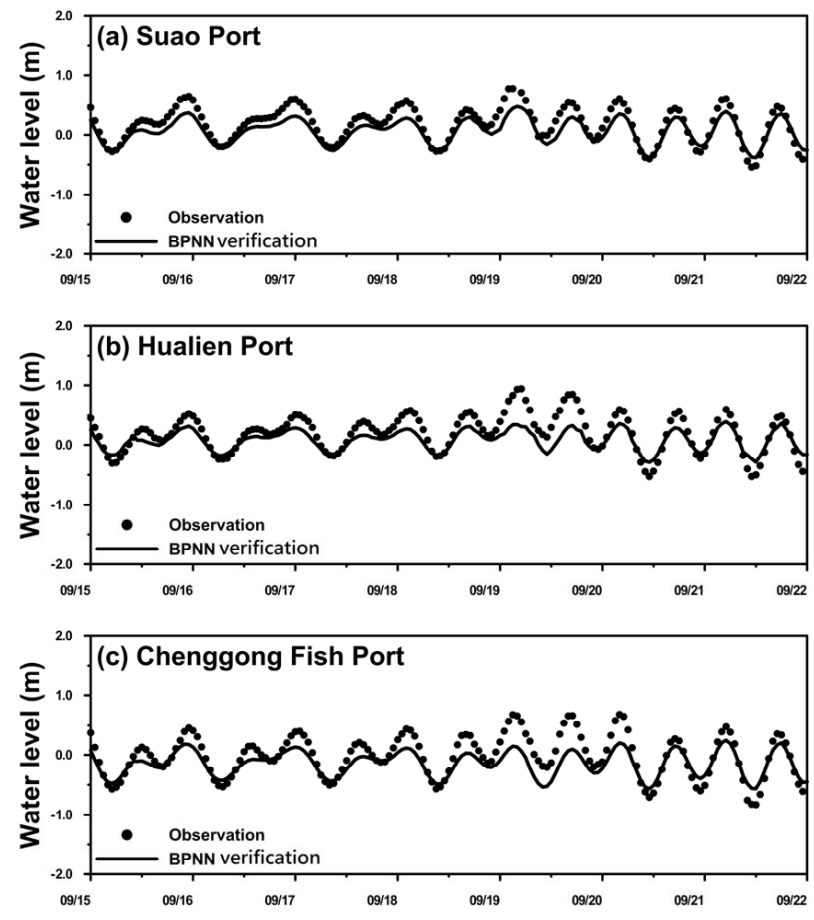

Fig. 10. Comparison of the observed (circle mark) and simulated (black line) water levels for the BPNN model in the verification phase at (a) Suao Port, (b) Hualien Port, and (c) Chenggong Fish Port during Typhoon Fanapi (2010).

Fish Port with the ANFIS model are less than those with the two-dimensional hydrodynamic model and BPNN model. The maximum PE, MAE, and RMSE values are $-13.77 \%$, $0.11 \mathrm{~m}$ and $0.16 \mathrm{~m}$, respectively, at Chenggong Fish Port for the ANFIS verification phase. The ANFIS model is successfully employed to improve the prediction of water level during typhoon events.

The degree to which uncertainty in the prediction of tidal elevations may influence the prediction of surge through tide-surge interactions is of interest. Formal solutions for the propagation of an externally forced tide and surge into an estuary of uniform section were developed by
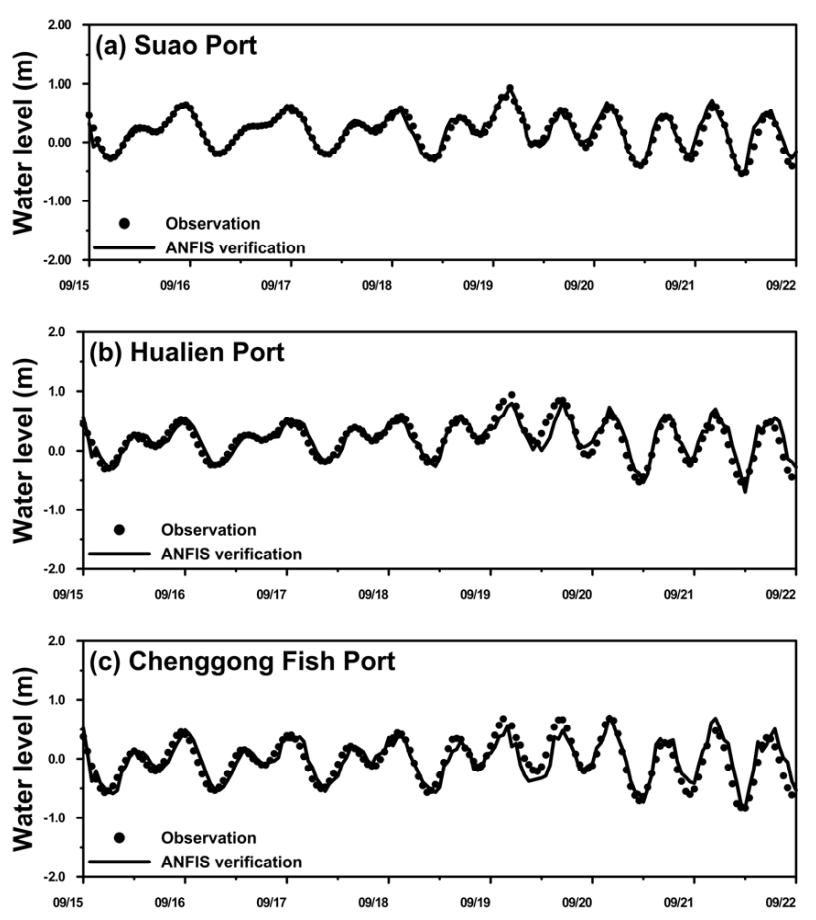

Fig. 11. Comparison of the observed (circle mark) and simulated (black line) water levels for the ANFIS model in the verification phase at (a) Suao Port, (b) Hualien Port, and (c) Chenggong Fish Port during Typhoon Fanapi (2010).

Proudman $(1955,1957)$. He concluded that there are effects of shallow water and bottom friction on the timing and magnitude of high water, both for standing wave and progressive wave. Rossiter (1961) assumed idealized surge with diurnal periodicity and showed how a negative surge would retard tidal propagation, whereas a positive surge would advance high water through a combination of depth affecting the wave propagation speed and depth-dependent frictional terms in the equation of motion. Prandle and Wolf (1978) reported tide-surge interactions resulting in a decrease of the peak surge elevation and that the effect can be localized, increasing in direct propagation to surge height and tidal range. Wolf (1981) used a one-dimensional analytical 
model to demonstrate that the shallow water effect becomes dominant over quadratic friction for tidal amplitudes in excess of $3 \mathrm{~m}$ and in depth of $10 \mathrm{~m}$ or less.

Recently, Bernier and Thompson (2006) performed a twodimensional barotropic ocean model in the northwest Atlantic and adjacent shelf seas and showed that the nonlinear parameterization of bottom stress is the principle contributor to their model tide-surge interaction off east coast of Canada and northeastern United States. Horsburgh and Wilson (2007) developed a simple mathematical explanation for surge clustering on the rising tide and proved that because of the interaction of the two signals, the peak of large surge events would typically avoid the high tide period by $3-5 \mathrm{~h}$, with a secondary clustering within $1-2 \mathrm{~h}$. Phase shifts occur in both the tide and the surge signals because of tidesurge interactions. This is because both signals are shallow water waves whose phase speed is given as $(g h)^{1 / 2}$ where $h$ is the depth and $g$ is the acceleration due to gravity. Zhang et al. (2010) studied the tide-surge interactions in the Taiwan Strait using the numerical model, NCTSM (two-way Nested Coupled Tide-Surge Model). They showed that the processes leading to the alteration of water elevations due to the tidesurge interactions can be classified into three nonlinear effects: nonlinear advective effects, nonlinear bottom stress effects, and nonlinear shallow water effects.

Ippen (1966) classified water waves according to the ratio of water depth $(h)$ to the wavelength $(L)$. The ratio $h / L$ is called the "relative depth". For the small amplitude wave theory, the equations expressing the various wave characteristics are greatly simplified; the ratio $h / L$ lies within certain ranges. If the relative depth is below $1 / 20$, then the depth is small in comparison with the wavelength and the waves are termed "shallow water" waves (the term "long wave"); if the ratio is greater than $1 / 2$, the waves are called "deep water" waves (also "short waves"). For $1 / 20<h / L<1 / 2$, the waves are called "intermediate depth" waves, and in this range the wave equations do not simplify. However, in a large number circumstances the waves are either in the "shallow water" or "depth water" category. The propagation of tides is attributed to long wave which has longer wavelength compared with the shorter (high-frequency) surge components. The wave propagation is quite different depending on the localized nature of tide-surge interaction in shallow water zones.

Tide-surge interactions are one of the most important problems in the study and affect the prediction of storm surges. However the study of tide-surge interactions is beyond the scope of present research. In the future work, the complex characteristics of tide-surge interactions off east coast of Taiwan can be investigated with the numerical model and dynamic analysis.

\section{Conclusions}

A high-resolution, two-dimensional, hydrodynamic model (ADCIRC), which uses an unstructured grid, was applied to simulate the astronomical tide level and storm surge height. To save computational time and fit the coastline boundary, coarse grids were generated in coastal seas, whereas fine grids were used in shallow areas close to the coastline. A global ocean tidal model developed by Oregon State University, the TOPEX/Poseidon Global Inverse Solution (TPXO), is used to specify the open boundaries of ADCIRC for simulating tidal propagation in the sea. A cyclone model is used for the meteorological conditions. Five typhoon events, Typhoon Mindulle (2004), Typhoon Sepat (2007), and Typhoon Kalmaegi (2008), Typhoon Funfwoug (2008) and Typhoon Fanapi (2010), were used for model calibration and verification. To determine the performance of the hydrodynamic model and the ANN model, three criteria (i.e. the peak error, $\mathrm{PE}$; mean absolute error, MAE; and the root-mean-square error, RMSE) were adopted to evaluate the model results and the observational data.

We found that the hydrodynamic model can satisfactorily reproduce the astronomical tide level but fails to mimic the storm surge height for the model calibration and verification phase. Therefore, the ANN model including the BPNN model and the ANFIS model was applied to amend the poor predictions in storm surge height during typhoon events with the two-dimensional hydrodynamic model. The simulation results reveal that the BPNN model can reproduce the astronomical tide but fails to improve the prediction of water level during storm surge. Moreover, the ANFIS model predicts both the astronomical tide level and the surge height and shows the lowest PE, MAE, and RMSE values at Suao Port, Hualien Port, and Chenggong Fish Port compared to the simulated results with the two-dimensional hydrodynamic model and the BPNN model. This study asserts that the ANFIS technique can be successfully employed in the prediction of water levels along the east coast of Taiwan during typhoon events.

In complex near shore regions, a high degree of uncertainty in predicted tides may induce uncertainty in the surge if tide-surge interactions are significant. The coasts in Taiwan have complex topography and bathymetry. Therefore, the tide-surge interactions off east coast of Taiwan will be studied with hydrodynamic modeling and dynamic analysis in the future.

Acknowledgements. The project was funded by the National Science Council, Taiwan, grant Nos. NSC 98-2625-M-239-001, 99-2625-M-239-001, 100-2625-M-239-001 and International Research-Intensive Centers of Excellence (I-RICE) in TaiwanSupercomputing Research Center, under grant No. NSC 103-2911I-006-301. The authors would like to express their appreciation to the Taiwan Center Weather Bureau for providing the observational data. Appreciation and thanks are also given to two reviewers for their constructive comments and suggestions to improve this paper. 
Edited by: S. Tinti

Reviewed by: D. Prandle and one anonymous referee

\section{References}

Bajo, M. and Umgiesser, G.: Storm surge forecast through a combination of dynamic and neural network models, Ocean Model., 33, 1-9, 2010.

Bernier, N. B. and Thopmson, K. R.: Predicting the frequency of storm surges and extreme sea levels in the northwest Atlantic, J. Geophys. Res., 111, C10009, doi:10.1029/2005JC003168, 2006.

De Oliveira, M. M. F., Ebecken, N. F. F., De Oliveira, J. L. F., and De Azevedo Santos, I.: Neural network model to predict a storm surge, J. Appl. Meteorol. Climatol., 48, 143-155, 2009.

Dietsche, D., Hagen, S. C., and Bacopoulos, P.: Storm surge simulation for Hurricane Hugo (1989): on the significance of inundation areas, J. Waterw. Port Coast. Ocean Eng., 133, 183-191, 2007.

Egbert, G. D. and Erofeeva, S. Y.: Efficient inverse modeling of barotropic ocean tides, Journal of Atmos. Ocean. Technol., 19, 183-204, 2002.

Egbert, G. D., Bennett, A. F., and Foreman, M. G.: TOPEX/POSEIDON tides estimated using a global inverse model, J. Geophys. Res., 99, 24821-24852, 1994.

Etala, P.: Dynamic issues in the SE South America storm surge modeling, Nat. Hazards, 51, 79-95, 2009.

Filippo, A., Torres Jr., A. R., Kjerfve, B., and Monat, A.: Application of artificial neural network (ANN) to improve forecasting of sea level, Ocean Coast. Manage., 55, 101-110, 2012.

Fletcher, R. and Powell, M. J. D.: A rapid convergent descent method for minimization, Comput. J., 6, 163-168, 1963.

Graham, H. E. and Nunn, D. E.: Meteorological conditions pertinent to standard project hurricane, Atlantic and Gulf Coasts of United States, National Hurricane Research Project, Report No. 3, US Weather Service, 1959.

Grenier, R. R., Luettich, R. A., and Westerink, J. J.: A comparison of the nonlinear frictional characteristics of two-dimensional and three-dimensional models of a shallow water tidal embayment, J. Geophys. Res., 100, 13719-13735, 1995.

Herman, A., Kaiser, R., and Niemeyer, H. D.: Modelling of a medium-term dynamics in a shallow tidal sea, based on combined physical neural network methods, Ocean Model., 17, 277299, 2007.

Horsburgh, K. J. and Wilson, C.: Tide-surge interaction and its role in the distribution of surge residuals in the North Sea, J. Geophys. Res., 112, C08003, doi:10.1029/2006JC004033, 2007.

Ippen, A. T.: Estuary and coastline hydrodynamics, McGraw-Hill Inc., 1-92, 1966.

Jang, J. S. R.: 1 ANFIS: adaptive-network-based fuzzy inference system, IEEE Trans. Syst. Man Cybern., Part A-Syst. Hum., 23, 665-685, 1993.

Jelesnianski, C. P.: A numerical calculation of storm tides induced by a tropical storm impinging on a continental shelf, Mon. Weather Rev., 93, 343-358, 1965.

Kolar, R. L. and Gray, W. G.: Shallow water modeling in small water bodies, edited by Gambolati, G., Computational Methods in Surface Hydrology, WIT Press, Billerica, Massachusetts, 149$155,1990$.
Kolar, R. L., Gray, W. G., Westerink, J. J., and Luettich, R. A.: Shallow water modeling in spherical coordinates-equation formulation, numerical implementation and application, J. Hydraul. Res., 32, 3-24, 1994.

Luettich, R. A., Westerink, J. J., and Scheffner, N. W.: ADCIRC: An advanced three-dimensional circulation model for shelves, coasts, and estuaries, Report I: theory and methodology of ADCIRC-2DDI and ADCIRC-3DL, US Army Corps of Engineers, Technical Report DRP-92-6, 1992.

Nayak, P. C., Sudheer, K. P., Ragan, D. M., and Ramasastri, K. S.: A neuro fuzzy computing technique for modeling hydrological time series, J. Hydrol., 291, 52-66, 2004.

Prandle, D. and Wolf, J.: The interaction of surge and tide in the North Sea and River Thames, Geophys. J. Astron. Soc., 55, 203 216, 1978.

Proudman, J.: The propagation of tide and surge in an estuary, Proc. R. Soc. Lond., A231, 8-24, 1955.

Proudman, J.: Oscillations of tide and surge in an estuary of finite length, J. Fluid Mech., 2, 371-381, 1957.

Rego, J. L. and Li, C.: Storm surge propagation in Galveston Bay during Hurricane Ike, J. Mar. Syst., 82, 265-279, 2010.

Rossiter, J. R.: Interaction between tide and surge in the Thames, Geophys. J. R. Astron. Soc., 6, 29-53, 1961.

Rumelhart, D. E., Hinton, G. E., and Williams, R. J.: Learning representations by back-propagating errors, Nature, 323, 533-536, 1986.

Shen, J., Wang, H. V., Sisson, M., and Gong, W.: Storm tide simulation in the Chesapeake Bay using an unstructured grid model, Estuar. Coast. Shelf Sci., 68, 1-16, 2006.

Takagi, T. and Sugeno, M.: Fuzzy identification of systems and its applications to modeling and control, IEEE Trans. Syst. Man Cybern., 15, 116-132, 1985.

Westerink, J. J., Luettich, R. A., and Muccino, J. C.: Modeling tides in the Western North Atlantic using unstructured graded grids, Tellus Ser. A-Dyn. Meteorol. Oceanol., 46, 178-199, 1994.

Wolf, J: Surge-tide interaction in the North Sea and River Thames, Floods die to High Winds and Tides, edited by: Peregrine, D. H., Elsevier, New York, 75-94, 1981.

Xia, M., Xia, L., Pietrafesa, L. J., and Peng, M.: A numerical study of storm surge in the Cape Fear River Estuary and adjacent coast, J. Coast. Res., 24, 159-167, 2008.

You, S. H. and Seo, J. W.: Storm surge prediction using an artificial neural network model and cluster analysis, Nat. Hazards, 51, 97114, 2009.

You, S. H., Lee, W. J., and Moon, K. S.: Comparison of storm surge/tide predictions between a 2-D operational forecast system, the regional tide/storm surge model (RTSM), and the 3-D regional ocean modeling system (ROMS), Ocean Dyn., 60, 443459, 2010.

Young, I. R. and Sobey, R. J.: The numerical prediction of tropical cyclone wind-waves, Department of Civil and Systems Engineering, James Cook University of North Queensland, Townville, Research Bulletin No. CS20, 1981.

Zhang, W., Shi, F., Hong, H., Shang, S., and Kirby, J.: Tide-surge intensified by the Taiwan Strait, J. Geophys. Res. 115, C06012, doi:10.1029/2009JC005762, 2010. 